\title{
Apicidin inhibits cell growth by downregulating IGF-1R in salivary mucoepidermoid carcinoma cells
}

\author{
MEE-YOUNG AHN ${ }^{1}$, JI-WEE AHN ${ }^{1}$, HYUNG-SIK KIM $^{2}$, JUN LEE $^{3}$ and JUNG-HOON YOON ${ }^{1}$ \\ ${ }^{1}$ Department of Oral and Maxillofacial Pathology, College of Dentistry, Wonkwang Bone Regeneration Research Institute, \\ Daejeon Dental Hospital, Wonkwang University, Daejeon 302-120; ${ }^{2}$ Division of Toxicology, School of Pharmacy, \\ Sungkyunkwan University, Suwon 440-746; ${ }^{3}$ Department of Oral and Maxillofacial Surgery, College of Dentistry, \\ Wonkwang Bone Regeneration Research Institute, Daejeon Dental Hospital, Wonkwang University, \\ Daejeon 302-120, Republic of Korea
}

Received October 6, 2014; Accepted January 12, 2015

DOI: $10.3892 / o r .2015 .3776$

\begin{abstract}
Inhibition of histone deacetylases (HDACs) has emerged as a new target for cancer therapies. The present study examined the antitumor effect and molecular mechanism of the HDAC inhibitor apicidin in YD-15 human salivary mucoepidermoid carcinoma (MEC) cells. The cells were treated with apicidin and cell death was quantified using an MTT assay. Apoptosis and autophagy were measured using flow cytometry, immunoblot analysis and cell staining. Regulation of the signaling pathways was monitored using immunoblot analysis and co-treatment with specific inhibitors. Insulin-like growth factor 1 receptor (IGF-1R) was knocked down using specific siRNA. Apicidin significantly inhibited the proliferation of MEC cells. Apicidin also induced apoptosis through the inactivation of extracellular signal-regulated kinase (ERK) and AKT/mTOR signaling and activation of c-Jun NH2-terminal kinase (JNK), whereas apicidin promoted autophagy through inactivation of the AKT/mTOR signaling. These effects may be mediated by the inhibition of IGF-1R, an upstream regulator of MAPK and AKT/mTOR pathways. These results suggested that apicidin is an attractive chemotherapeutic agent against salivary MEC and may be a good candidate for targeting IGF-1R for cancer therapies.
\end{abstract}

\section{Introduction}

Several diseases, especially cancer, are caused by aberrant epigenetic alterations in addition to genetic mutations $(1,2)$. Histone acetylation, an example of epigenetic regulation, is a process that is under intensive study since it regulates gene transcription. Histone deacetylases (HDAC), which regulate histone

Correspondence to: Professor Jung-Hoon Yoon, Department of Oral and Maxillofacial Pathology, College of Dentistry, Daejeon Dental Hospital, Wonkwang University, Daejeon 302-120, Republic of Korea E-mail: opathyoon@wku.ac.kr

Key words: apicidin, HDAC inhibitor, IGF-1R, salivary mucoepidermoid carcinoma, apoptosis, autophagy acetylation, are often inappropriately expressed in cancer cells, which lead to the silencing of tumor-suppressor genes or the activation of oncogenes (3). HDAC inhibition abrogates epigenetic silencing commonly observed in cancer, and a number of HDAC inhibitors have been developed for cancer treatment (4). Several HDAC inhibitors have been identified through synthetic or natural sources and separated into several structurally distinct classes: short chain fatty, and hydroxamic acids, benzamides, cyclic tetrapeptides, and electrophilic ketones. Members of those classes show substantially different potency and target specificity $(3,5)$. Apicidin, a cyclic peptide HDAC inhibitor, was the first reported reversible inhibitor of the in vitro development of apicomplexan parasites and was later shown to have antiproliferative and cyto-differentiation activity in mammalian cells $(6,7)$. Apicidin has been shown to exhibit antitumor activity in several human cancer cells. Previous studies reported that apicidin induced apoptosis and cell cycle arrest in ovarian, endometrial and breast cancer cells (8-10). Nevertheless, the precise molecular mechanism of cell death effect by apicidin remains unclear and, to the best of our knowledge, has not been previously examined in salivary mucoepidermoid carcinoma (MEC), the most common malignant salivary tumor corresponding to $34 \%$ of all salivary malignancies (11).

Cell death occurs through at least three morphologically distinct subroutines, known as apoptosis, necrosis and autophagy (12). Apoptosis is a well-defined, programmed mechanism that allows cells to commit suicide and is the major type of cell death induced by various antitumor drugs including HDAC inhibitors $(3,5,13)$. Autophagy plays an important homeostatic role, mediating the removal of dysfunctional or damaged organelles, which are digested and recycled for cellular metabolic needs (14). Autophagy recently attracted considerable attention in cancer research. However, present autophagy studies are often viewed as confounding, because of its association with apparently contradictory roles, such as survival and cell death, depending on the model used $(15,16)$. Apoptosis and necrosis are irreversible forms of cell death, whereas autophagy leads to cell death or paradoxically allows cells to escape cell death (17). In recent studies autophagy has been identified in tumor cell lines treated with HDAC inhibitors. However, whether autophagy contributes 
to the anticancer activity of HDAC inhibitors remains to be clarified $(16,18)$. Previous studies reported that apicidin induces autophagy in oral squamous cell carcinoma (OSCC) cells, however, the molecular mechanism of apicidin has yet to be thoroughly identified (18). The present study evaluated the antitumor effect of apicidin and examined whether the molecular mechanisms of apicidin induce cell death in human salivary MEC cells.

\section{Materials and methods}

Chemicals and cell culture. Apicidin [Cyclo (N-O-methylL-tryptophanyl-L-isoleucinyl-D-pipecolinyl-L-2-amino-8oxo decanoyl)] was purchased from Sigma-Aldrich (St. Louis, MO, USA). Apicidin was dissolved in sterile dimethyl sulfoxide (DMSO; Sigma-Aldrich) to generate a $5 \mathrm{mM}$ stock solution, and stored at $-80^{\circ} \mathrm{C}$. Subsequent dilutions were made in RPMI-1640 (Gibco-BRL, Rockville, MD, USA). Caspase pan-inhibitor z-VAD-fmk was purchased from MBL Co. (Woburn, MA, USA). Extracellular signal-regulated kinase (ERK) inhibitor U0126 was purchased from Cell Signaling Technology, Inc. (Danvers, MA, USA). c-Jun NH2-terminal kinase (JNK) inhibitor SP600125 and PI3K/AKT inhibitor LY294002 were purchased from Cayman Chemical Co. (Ann Arbor, Michigan, USA). All other chemicals were purchased from Sigma-Aldrich.

The YD-15 human salivary MEC cells were purchased from the Korea Cell Line Bank (KCLB, Seoul, Korea) (19). The cells were maintained as monolayers at $37^{\circ} \mathrm{C}$ in an atmosphere containing $5 \% \mathrm{CO}_{2}$ /air in RPMI-1640 containing $10 \%$ heat-inactivated fetal bovine serum (FBS; Gibco-BRL, Gaithersburg, MD, USA) and $1 \%$ penicillin/streptomycin (Gibco-BRL).

MTT assay. The cells were grown in 96-well plates at a density of $1 \times 10^{4}$ cells/well. The cells were allowed to attach for $24 \mathrm{~h}$, and were then exposed to apicidin. At the end of the treatment period, $20 \mu \mathrm{l}$ of 3-(4,5-dimethylthiazol-2-yl)-2,5-diphenyltetrazolium bromide (MTT; $5 \mathrm{mg} / \mathrm{ml}$, Sigma-Aldrich) reagent was added to each well. After $4 \mathrm{~h}$ incubation in the dark at $37^{\circ} \mathrm{C}$, the supernatant was aspirated and formazan crystals were dissolved in $100 \mu \mathrm{l}$ of DMSO at $37^{\circ} \mathrm{C}$ for 10 min with gentle agitation. The absorbance per well was measured at $540 \mathrm{~nm}$ using a VersaMax microplate reader (Molecular Devices Corp. Sunnyvale, CA, USA).

HDAC activity. The HDAC enzyme activity was measured using a fluorogenic HDAC Assay kit purchased from BD Biosciences (San Diego, CA, USA) according to the manufacturer's instructions. Briefly, the cell lysates were incubated with vehicle or various concentrations of apicidin and SAHA at $37^{\circ} \mathrm{C}$ for $30 \mathrm{~min}$ in the presence of an HDAC fluorimetric substrate. The HDAC assay developer, which produces a fluorophore in the reaction mixture, was added, and the fluorescence was measured using Victor3 (PerkinElmer, Waltham, MA, USA) with excitation at $360 \mathrm{~nm}$ and emission at $460 \mathrm{~nm}$. The measured activity was assessed using GraphPad Prism (GraphPad Software, San Diego, CA, USA).

Trypan blue exclusion assay. The trypan blue exclusion assay was based on the ability of viable cells to exclude the dye.
Five minutes after $0.4 \%$ trypan blue was added to the cells, they were loaded into a hematocytometer and counted for the dye uptake. The number of viable cells was calculated as a percentage of the total cell population.

Western blot analysis. The cells were seeded in 6-well plates and exposed to the indicated concentrations (1.0 and $5.0 \mu \mathrm{M})$ of apicidin. After treatment, the cells were harvested using lysis buffer (Pro-Prep, Daejeon, Intron, Korea). Protein concentrations were measured using the Protein Assay kit (Bio-Rad, Hercules, CA, USA) according to the manufacturer's instructions. Total protein was then separated using 10-15\% sodium dodecyl sulfate (SDS)-polyacrylamide gels and transferred to polyvinylidene difluoride (PVDF) membrane (NEN Life Science Products, Boston, MA, USA). The membranes were probed sequentially with antibodies against acetylated histone $\mathrm{H} 3$, acetylated histone $\mathrm{H} 4$ (Upstate, Lake Placid, NY, USA), Bax, cytochrome $c$, cleaved caspase-9, -7 and -3, procaspase 3, poly ADP ribose polymerase (PARP), light change $3 \mathrm{~B}$ (LC3B), insulin-like growth factor 1 receptor (IGF-1R), ERK, p-ERK, JNK, p-JNK, p38, p-p38, AKT, p-AKT, p-mTOR (Cell Signaling Technology Inc.), p62, and Actin (Santa Cruz Biotechnology, Inc., CA, USA) at $4^{\circ} \mathrm{C}$ for overnight. The blots were developed using an enhanced chemiluminescence (ECL) kit (Amersham Biosciences, Buckinghamshire, UK). The protein levels were normalized by a comparison with the actin levels.

Flow cytometric analysis. The cells were stained using an Alexa Fluor ${ }^{\circledR} 488$ Annexin V/Dead Cell apoptosis kit (Invitrogen, Eugene, Oregon, USA) for apoptosis analysis according to the manufacturer's instructions. Data acquisition and analysis were carried out by a Cell Lab Quanta ${ }^{\mathrm{TM}} \mathrm{SC}$ flow cytometer (Beckman Coulter Inc., Miami, FL, USA) and software.

Detection of acidic vesicular organelles. Autophagy is characterized by the formation and promotion of acidic vesicular organelles (AVOs). To detect the development of AVOs, the cells were stained with acridine orange as previously described (20). In acridine orange-stained cells, the cytoplasm and nucleus show bright green fluorescence, whereas the acidic compartments exhibit bright red fluorescence (autophagic cells). Briefly, the treated cells were stained with acridine orange ( $1 \mu \mathrm{g} / \mathrm{ml}$, Sigma-Aldrich) for $15 \mathrm{~min}$. The samples were observed under fluorescence microscopy (Olympus, Centre Valley, PA, USA). To quantify the development of AVOs, the stained cells were analyzed by FACScan flow cytometer (Beckman Coulter Inc.) with CellQuest software.

Monodansylcadaverine staining. The autofluorescent agent monodansylcadaverine (MDC; Sigma-Aldrich) was used to evaluate the abundance of autophagic vacuoles in cells. Cells were stained with $\mathrm{MDC}$ at a final concentration of $0.05 \mathrm{mmol} / 1$, for $10 \mathrm{~min}$ at $37^{\circ} \mathrm{C}$. The cells were washed with phosphate-buffered saline and observed under fluorescence microscopy (Olympus).

siRNA transfection. The siRNA oligonucleotides for IGF-1R were synthesized by Bioneer (Daejeon, Korea), and the non-targeting control siRNA (NC. siRNA) was used as the 

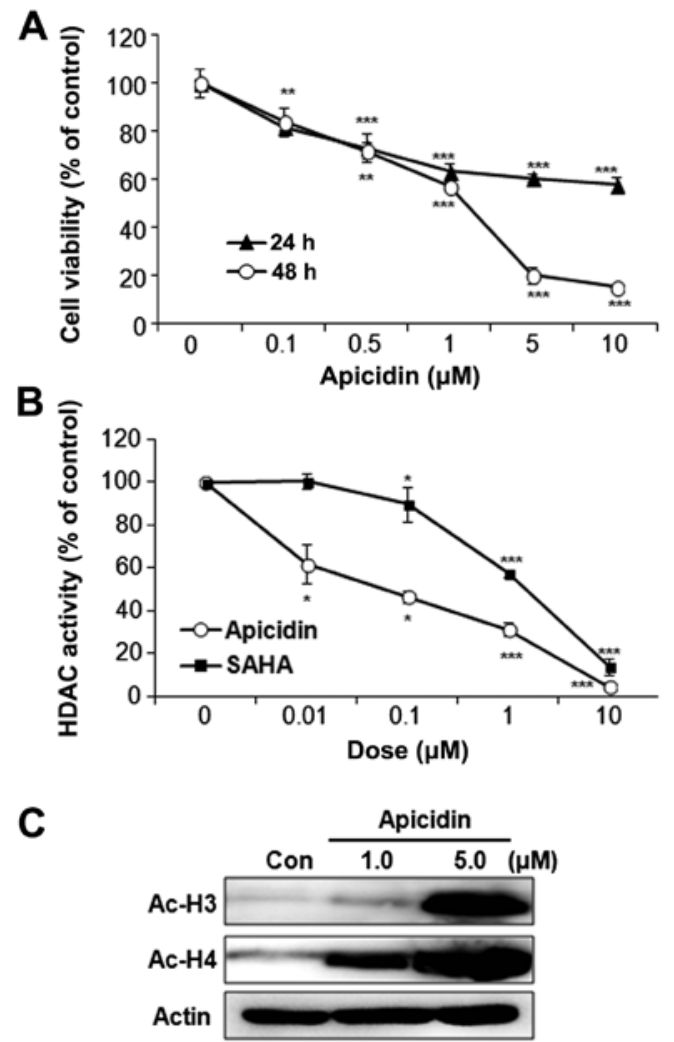

Figure 1. Effect of apicidin on the proliferation and HDAC activity in YD-15 human salivary MEC cells. (A) The cells were treated with various concentrations (0.1-10.0 $\mu \mathrm{M})$ of apicidin for $48 \mathrm{~h}$. The levels of cell proliferation were measured using an MTT assay. The percentage of viable cells was calculated as the ratio of treated cells to the control cells. (B) HDAC activity in HDAC inhibitor-treated YD-15 cells. The data are presented as the mean $\pm \mathrm{SD}$ of three independent experiments. ${ }^{*} \mathrm{P}<0.05,{ }^{* *} \mathrm{P}<0.01$ and ${ }^{* * * *} \mathrm{P}<0.001$ compared to the untreated control. (C) Immunoblot analysis of the levels of acetylation-associated proteins in YD-15 cells. The cells were treated with the indicated concentrations of apicidin for $48 \mathrm{~h}$. The total cell lysates were prepared and $30 \mu \mathrm{g}$ of the protein was subjected to SDS-PAGE followed by western blot analysis and chemiluminescent detection. Western blot analysis was performed using a series of antibodies and acetylated histone $\mathrm{H} 3$ and $\mathrm{H} 4$ levels were analyzed. The protein levels were normalized by a comparison with the actin levels. The representative bands from three independent experiments are shown. MEC, mucoepidermoid carcinoma; SDS, sodium dodecyl sulfate; HDAC, histone deacetylase.

negative control. The IGF-1R-siRNA sequence used was: sense, 5'-CGACAGGCCUCGUGUAUGA(UU)-3', and anti-sense, 5'-UCAUACACGAGGCCUGUCG(UU)-3' (21). The cells were transfected with $100 \mathrm{nM}$ siRNA for $24 \mathrm{~h}$ using Lipofectamine Plus reagent (Invitrogen). The cells were harvested for protein analysis 3 days after transfection. Western blot analysis was used to validate silencing of the protein expression.

Statistical analysis. Data are presented as the mean \pm SD of at least three individual experiments. Statistical comparisons between the groups were performed using a two-tailed Student's t-test (Excel, Microsoft). Statistical significance differences were set at $\mathrm{P}<0.05, \mathrm{P}<0.01$ and $\mathrm{P}<0.01$.

\section{Results}

Apicidin inhibits cell proliferation and HDAC activity. The effect of apicidin on MEC cell proliferation was first examined using the MTT assay. The cells were treated with various doses of apicidin until $48 \mathrm{~h}$. Apicidin significantly inhibited the cell proliferation of YD-15 cells in a dose- and time-dependent manner (Fig. 1A). Specifically, cell viability was markedly decreased when the cells were treated with $5.0 \mu \mathrm{M}$ apicidin for $48 \mathrm{~h}$. The enzyme activity and the expression of acetylated histones were next examined to determine the HDAC inhibitory effect by apicidin. The HDAC activity was markedly inhibited by apicidin in YD-15 cells, which reduced the HDAC activity more effectively as compared to SAHA, the first HDAC inhibitor to obtain FDA approval (Fig. 1B). In addition, apicidin significantly increased the levels of acetylated histone H3 and H4 (Fig. 1C).

Induction of apoptosis by apicidin. The apoptotic effect of apicidin was examined by flow cytometry and western blotting. Apicidin significantly increased the number of Annexin V-positive apoptotic cells in a dose-dependent manner (Fig. 2A). As shown in Fig. 2B, cytochrome $c$ levels were increased in the cytosol fraction, and Bax and cleaved PARP were also induced in YD-15 cells after apicidin treatment. Apicidin significantly induced the activation of caspase-9, -7 and -3 in YD-15 cells (Fig. 2C). These results showed that apicidin induced caspase-dependent apoptosis via the mitochondria-mediated intrinsic pathway in YD-15 MEC cells.

Induction of autophagy by apicidin. The levels of the microtubule-associated protein 1 LC3-II, a marker for autophagic vesicles and p62 as a marker for autophagic flux were determined to investigate the possibility of apicidin-inducing autophagy in YD-15 cells. As shown in Fig. 3A, the level of LC3B-II increased by apicidin treatment in a dose-dependent manner, whereas p62 was decreased. The autophagy response by apicidin was examined using acridine orange and MDC staining. Flow cytometry using acridine orange staining showed that apicidin induced the number of acidic vesicles (red signal) in a dose-dependent manner, as confirmed by fluorescence microscopy (Fig. 3B and C). MDC staining also showed increased AVOs in apicidin-treated cells compared to the control (Fig. 3C). These results showed that apicidin induced autophagy in YD-15 cells.

Function of autophagy and interaction between autophagy and apoptosis. To investigate the function of autophagy by apicidin and interaction between apoptosis and autophagy, YD-15 cells were co-treated with the specific autophagy inhibitor, chloroquine (CQ), and apicidin. Cell viability was examined using the Trypan blue exclusion assay. As shown in Fig. 4A, co-treatment with apicidin and CQ reduced cell viability compared to apicidin treatment alone. The levels of PARP and LC3B-II expression were examined by western blotting. Apicidin with CQ increased the levels of PARP cleavage and LC3B-II arrest by inhibiting autophagic degradation compared to apicidin-alone treatment (Fig. 4B). These results indicated that the inhibition of autophagy enhanced apicidin-induced apoptosis (Fig. 4B). Increased apoptosis by autophagy inhibition was confirmed by flow cytometry. The percentage of apoptotic cells was significantly increased in the co-treated cells compared to apicidin alone-treated cells (Fig. 4C). These results suggested that autophagy has a prosurvival function in YD-15 cells. 
A
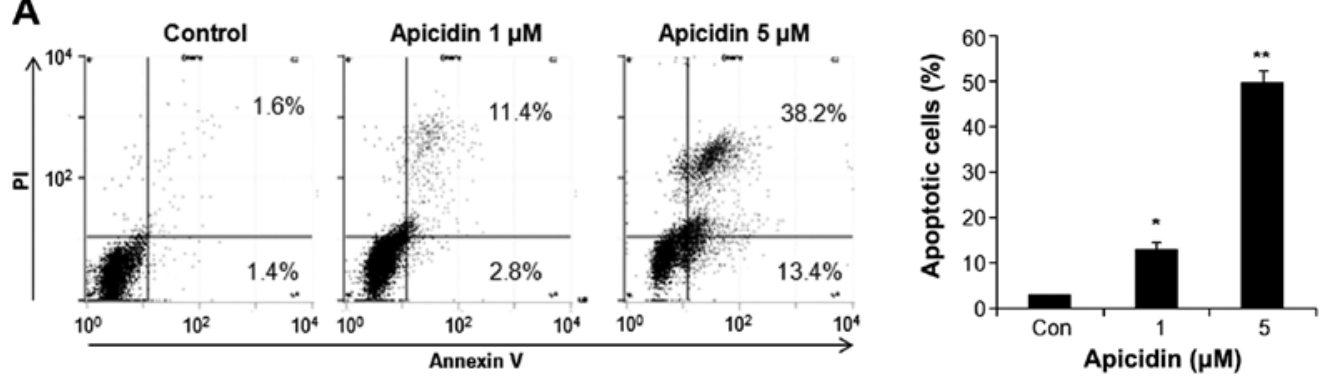

B

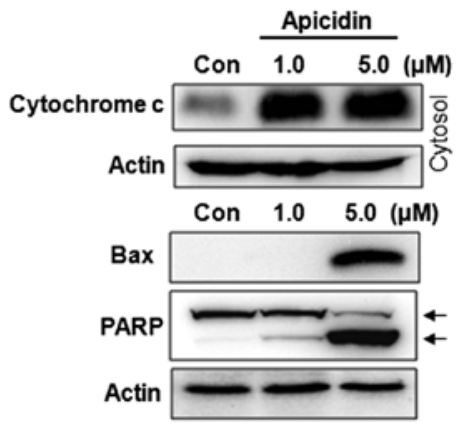

C

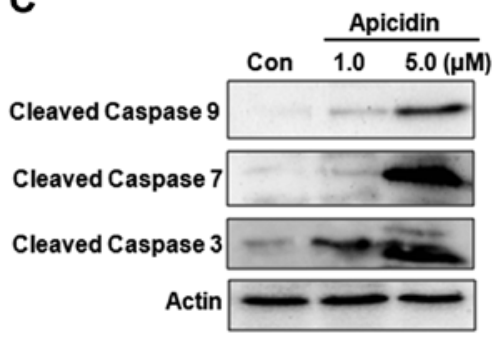

Figure 2. Apoptosis analysis of apicidin-treated YD-15 cells. (A) Annexin V/PI staining for apoptotic cells. Cells were incubated with apicidin for $48 \mathrm{~h}$. The apoptotic cells were detected using Alexa Fluor ${ }^{\circledast} 488$ Annexin V/Dead Cell apoptosis kit and the flow cytometry results are shown. The data are presented as the mean \pm SD of three independent experiments. ${ }^{*} \mathrm{P}<0.05,{ }^{* *} \mathrm{P}<0.01$ compared to the untreated control. (B and C) Expression of the apoptosis-associated proteins in YD-15 cells. The cells were treated with apicidin $(1.0$ and $5.0 \mu \mathrm{M})$ for $48 \mathrm{~h}$. Cell cytosolic extracts were subjected to western blot analysis using an antibody against cytochrome $c$. The total cell lysates were prepared and the protein was subjected to SDS-PAGE followed by western blot analysis and chemiluminescent detection. Western blot analysis was performed using a series of antibodies; Bax, PARP, cleaved caspase- $9,-7$ and -3 . The protein levels were normalized by a comparison with the actin levels. The representative bands from three independent experiments are shown. SDS, sodium dodecyl sulfate; PARP, poly ADP ribose polymerase; PI, propidium iodide.
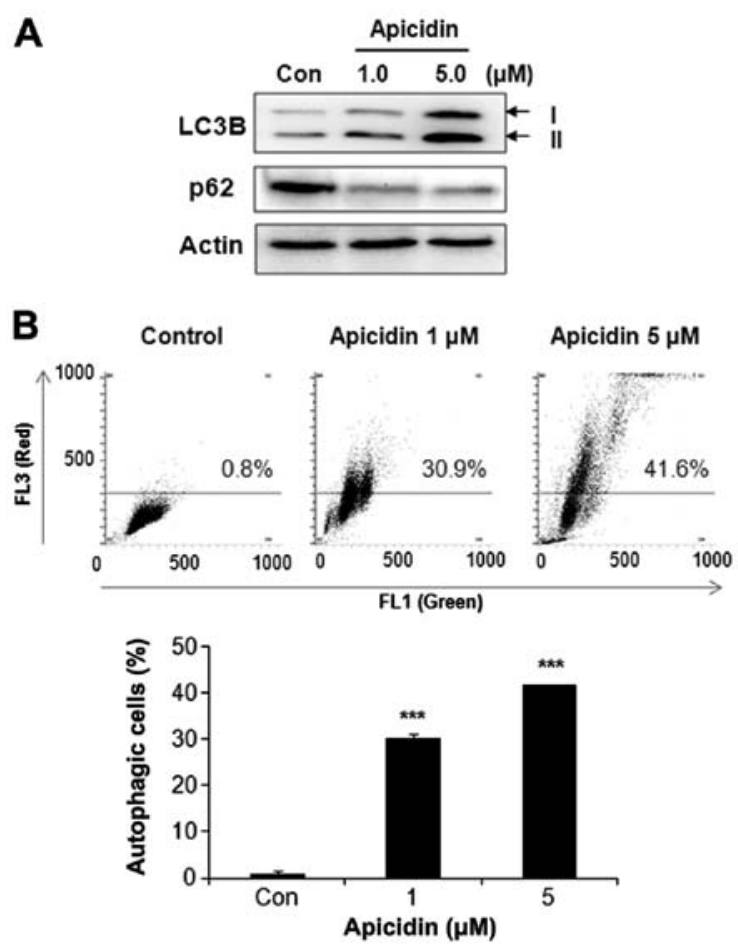

C
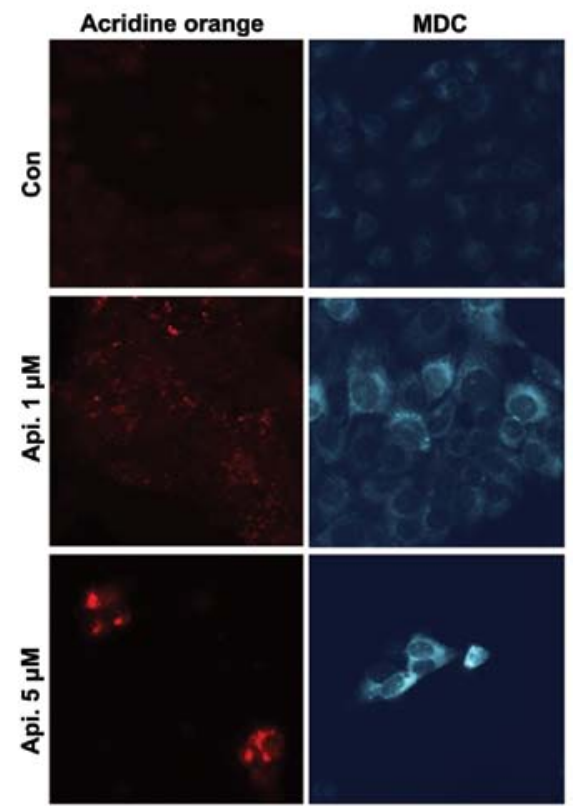

Figure 3. Autophagy detection in apicidin-treated YD-15 cells. (A) Expression of the autophagy-associated proteins in YD-15 cells. The cells were treated with apicidin $(1.0$ and $5.0 \mu \mathrm{M})$ for $48 \mathrm{~h}$. Cell lysates were subjected to western blot analysis using an antibody against LC3B and p62. The protein levels were normalized by a comparison with the actin levels. The representative bands from three independent experiments are shown. (B) Fluorescence-activated cell sorting analysis using acridine orange. The cells treated with apicidin for $48 \mathrm{~h}$ were stained with acridine orange $(1 \mu \mathrm{g} / \mathrm{ml})$ and subjected to flow cytometry. FL1, green color intensity; FL3, red color intensity. Top of the grid was considered as AVO. The data are presented as the mean \pm SD of three independent experiments. ${ }^{* * *} \mathrm{P}<0.001$ compared to the untreated control. (C) Acridine orange and monodansylcadaverine (MDC) staining. After apicidin treatment for $48 \mathrm{~h}$, the cells were incubated with MDC $(0.05 \mathrm{mmol} / \mathrm{l})$ for $10 \mathrm{~min}$ and stained with acridine orange $(1 \mu \mathrm{g} / \mathrm{ml})$, and then observed by fluorescence microscopy (magnification, x200). The representative results from three independent experiments are shown. LC3B, light change 3B. 


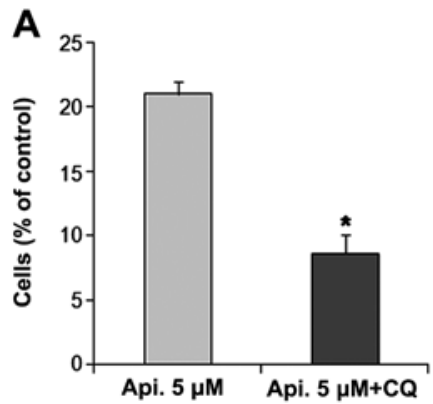

B

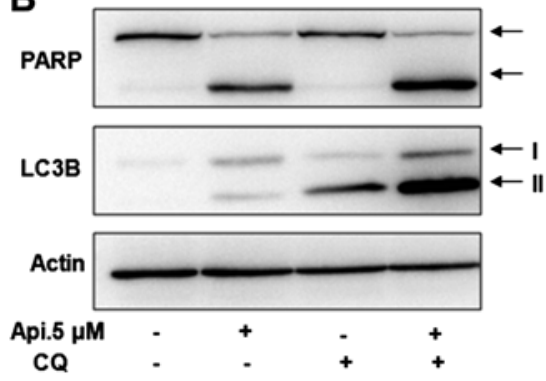

C
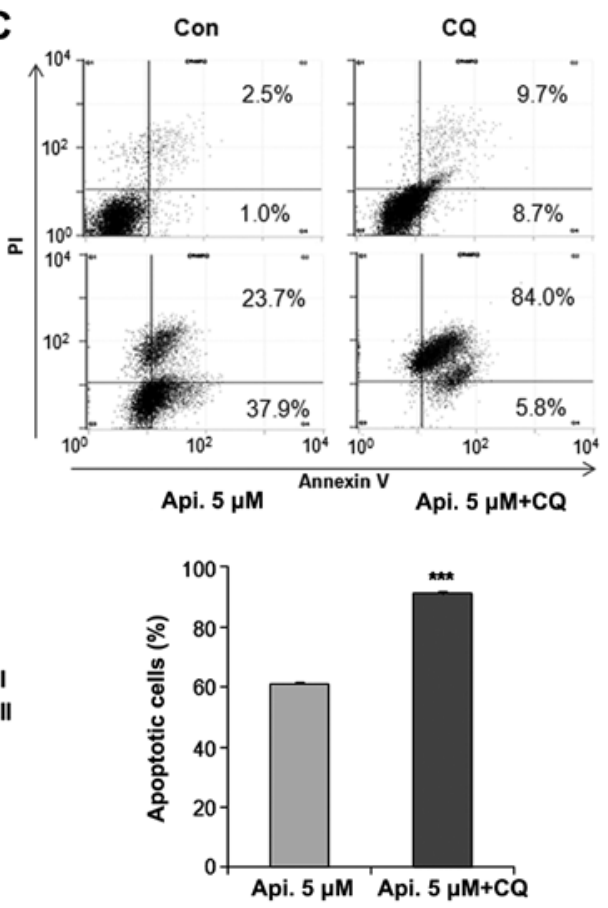

Figure 4. Autophagy inhibition enhances the proapoptotic effects of apicidin in YD-15 cells. The cells were treated with $5 \mu \mathrm{M}$ apicidin for $48 \mathrm{~h}$ in the absence or presence of CQ $(50 \mu \mathrm{mol} / \mathrm{l})$. (A) Cell viability was determined using a trypan blue exclusion assay after co-treatment with apicidin and CQ. (B) Western blot analysis was performed using PARP and LC3B antibodies in cells co-treated with $5 \mu \mathrm{M}$ apicidin with CQ. (C) Apoptosis was determined by flow cytometry for Annexin V-FITC and PI dual labeling. The population of apoptotic cells was calculated. The cells in the upper-right and lower-right quadrants are the apoptotic cells. The data are presented as the mean \pm SD of three independent experiments. ${ }^{*} \mathrm{P}<0.05,{ }^{* * * *} \mathrm{P}<0.001$ compared to the untreated control. CQ, chloroquine; $\mathrm{PI}$, propidium iodide; PARP, poly ADP ribose polymerase; LC3B, light change 3B.

Apicidin-induced apoptosis was oppositely inhibited by co-treatment with a caspase pan-inhibitor, z-VAD-fmk, and the induction of apoptosis and autophagy was observed. Apicidin with z-VAD-fmk increased the cell viability and reduced the levels of cleaved PARP and caspase-3 compared to apicidin-alone treatment (Fig. 5A and B). Reduced apoptosis by co-treatment was confirmed by flow cytometry (Fig. 5C). However, apicidin treatment with $\mathrm{z}$-VAD-fmk increased the level of LC3B-II expression and reduced p62 expression compared to apicidin treatment (Fig. 5B). These results showed that the inhibition of apoptosis enhanced apicidin-induced autophagy in YD-15 cells.

Regulation of MAPK and AKT/mTOR pathway by apicidin in $Y D-15$ cells. To evaluate the molecular mechanism of apoptosis or autophagy by apicidin, the regulation of MAPK and AKT/mTOR pathways was monitored in apicidin-treated cells. These signaling pathways are important in cell survival and proliferation events and are well-established mediators of the malignant phenotype. The MAPK family mainly consists of ERK, JNK, and p38 MAPK kinase members (22). The effects of apicidin on the activation of ERK, JNK and p38 MAP kinases were first examined by western blotting. Apicidin decreased the level of ERK phosphorylation and increased the level of JNK phosphorylation, but had no effect on the level of p38 phosphorylation (Fig. 6A). The phosphorylation of AKT and mTOR as the main downstream mediator of AKT was measured in apicidin-treated cells. Apicidin markedly inhibited the phosphorylation of AKT and mTOR in YD-15 cells (Fig. 6B). These results suggested that apicidin inhibited cell proliferation by regulating ERK, JNK and AKT/mTOR pathways.
Involvement of the MAPK and AKT/mTOR pathways in apicidin-induced apoptosis and autophagy. In order to determine which pathway is involved in apoptosis or autophagy in apicidin-treated YD-15 cells, the cells were co-treated with apicidin and specific MAPK or AKT/mTOR pathway kinase inhibitors, including U0126 (ERK inhibitor), SP600125 (JNK inhibitor) and LY294002 (PI3K/AKT inhibitor). As shown in Fig. 7A, enhanced ERK inhibition by apicidin with U0126 increased the cleaved PARP and decreased the cell viability of the co-treated cells compared to apicidin alone-treated cells. On the other hand, there were no significant differences in LC3B-II expression. The inhibition of apicidin-induced JNK phosphorylation by SP600125 reduced cleaved PARP, but not LC3B-II expression, and increased the cell viability in the co-treated cells compared to the apicidin-treated cells. This result suggested that the inhibition of ERK and induction of JNK signals by apicidin mediated apoptosis in YD-15 cells, but not autophagy. Enhanced AKT inhibition by apicidin with LY294002 induced the increase of cleaved PARP and the decrease of pro-caspase-3, indicating the induction of apoptosis. In addition, the level of LC3B-II expression was higher in the co-treated cells than the apicidin-treated cells (Fig. 7B). These results suggested that the inhibition of AKT/mTOR signal by apicidin mediated apoptosis and autophagy simultaneously in YD-15 cells. Induction of apoptosis and autophagy through enhanced AKT inhibition was confirmed by flow cytometric analysis using Annexin V/PI and acridine orange staining in co-treated YD-15 cells (Fig. 7C).

Inhibition of IGF-1R was associated with apoptosis and autophagy by apicidin. IGF-1R is commonly overexpressed 
A

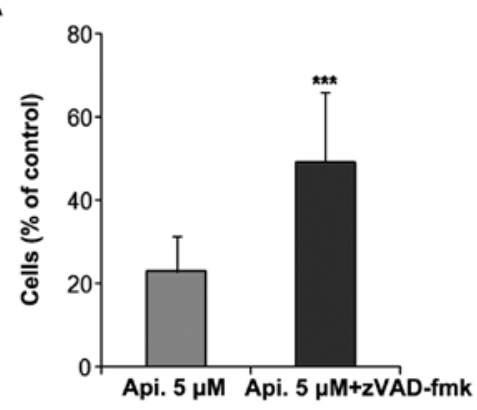

B

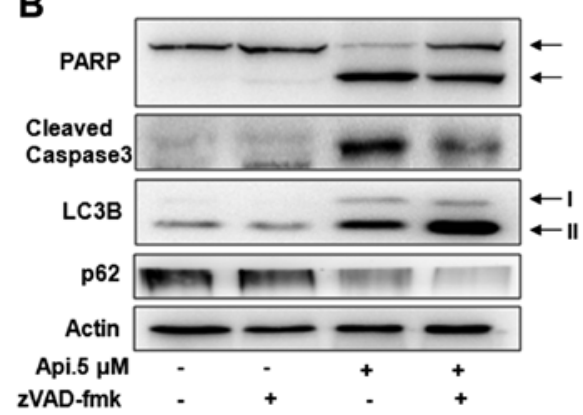

C
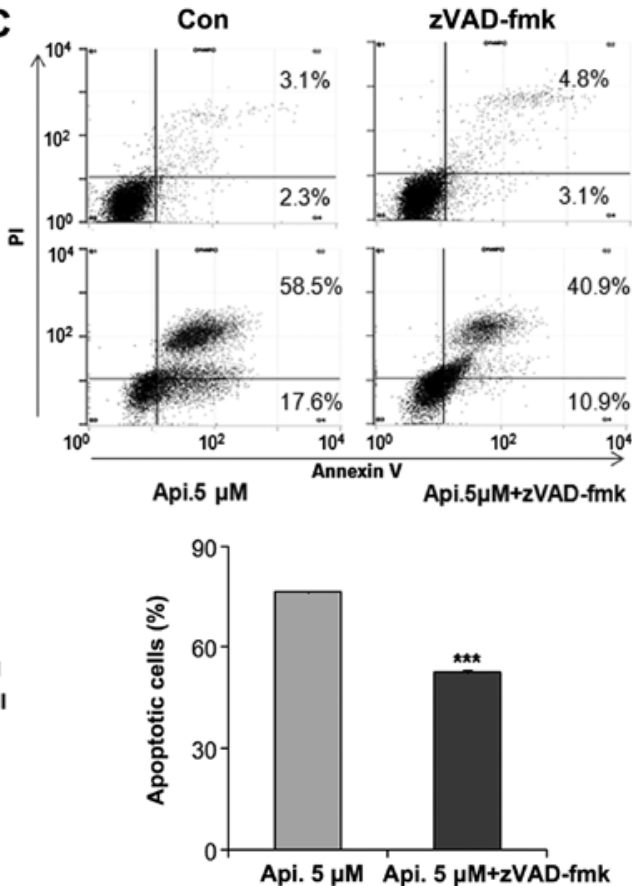

Figure 5. Apoptosis inhibition enhances the autophagic effects of apicidin in YD-15 cells. The cells were treated with $5 \mu \mathrm{M}$ apicidin for $48 \mathrm{~h}$ in the absence or presence of z-VAD-fmk $(20 \mu \mathrm{mol} / \mathrm{l})$. (A) Cell viability was determined using a trypan blue exclusion assay after co-treatment with apicidin and z-VAD-fmk. (B) Western blot analysis was performed using PARP, cleaved caspase-3, LC3B and p62 antibodies in cells co-treated with $5 \mu \mathrm{M}$ apicidin with z-VAD-fmk. (C) Apoptosis was determined by flow cytometry for Annexin V-FITC and PI dual labeling. The population of apoptotic cells was calculated. The cells in the upper-right and lower-right quadrants are the apoptotic cells. The data are presented as the mean \pm SD of three independent experiments. ${ }^{* * *} \mathrm{P}<0.001$ compared to the untreated control. PI, propidium iodide; PARP, poly ADP ribose polymerase; LC3B, light change 3B.

A

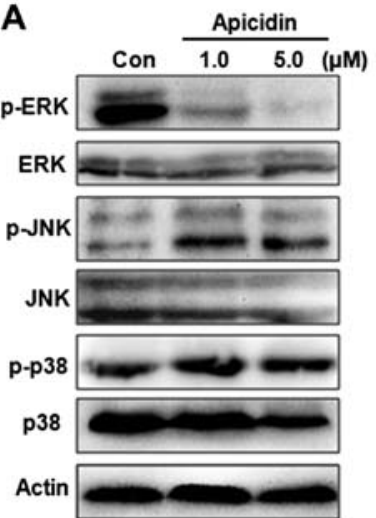

B

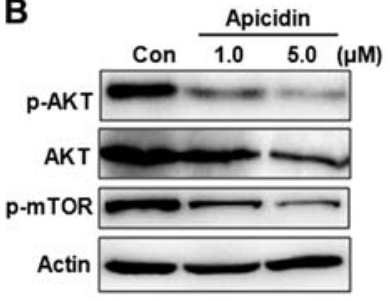

Figure 6. Expression of MAPKs and AKT/mTOR signals in apicidin-treated YD-15 cells. The cells were treated with apicidin 1.0 and $5.0 \mu \mathrm{M}$ for $48 \mathrm{~h}$ Cell lysates were subjected to western blot analysis using an antibody against (A) p-ERK, ERK, p-JNK, JNK, p-p38 and p38, and (B) p-AKT, AKT and p-mTOR. The protein levels were normalized by a comparison with the actin levels. The representative bands from three independent experiments are shown. ERK, extracellular signal-regulated kinase; JNK, c-Jun NH2-terminal kinase.

in cancer cells and stimulates two major downstream proliferating signaling pathways, the MAPK and AKT/mTOR pathways (23). We examined whether apicidin inhibited the expression of IGF-1R inducing the inhibition of MAPK and AKT/mTOR pathways in YD-15 cells. As shown in Fig. 8A, apicidin significantly inhibited the level of IGF-1R expression in a dose-dependent manner. To determine whether the inhibition of IGF-1R by apicidin was associated with the induction of apoptosis and autophagy, IGF-1R was knocked down by treatment with a specific IGF-1R siRNA (siIGF-1R)

in YD-15 cells. Knockdown of IGF-1R inhibited cell proliferation and induced Annexin V-positive apoptotic cells and AVO formation, as indicated by the autophagic marker by flow cytometric analysis (Fig. 8B and C). The expression levels of cleaved PARP and LC3B-II were also increased in siIGF-1Rtransfected YD-15 cells (Fig. 8D). These results showed that IGF-1R knockdown induced apoptosis and autophagy in YD-15 cells. We also examined whether the effect of IGF-1R knockdown on apoptosis and autophagy was associated with the regulation of ERK, JNK and AKT/mTOR signals by western blotting. As shown in Fig. 8E, knockdown of IGF-1R markedly inhibited ERK phosphorylation and induced slight JNK phosphorylation in YD-15 cells. The inhibition of AKT and mTOR phosphorlyation was also observed in siIGF-1Rtreated cells. These effects of IGF-1R knockdown in siIGF-1Rtransfected YD-15 cells showed a similar pattern to that observed in apicidin-treated YD-15 cells. Therefore, the inhibition of IGF-1R in YD-15 cells by apicidin was strongly mediated by the induction of apoptosis and autophagy through regulation of the ERK, JNK and AKT/mTOR pathways.

\section{Discussion}

Salivary gland cancers account for $\sim 5 \%$ of all head and neck malignancies (24). MEC is the most common primary malignancy in the salivary gland, however, few clinical trial data have addressed the role of systemic therapy specific to this type of tumor (25). HDAC inhibitors are a relatively new class of anticancer agents that play an important role in inducing apoptosis, and cell cycle arrest in several cancer cells $(1,8,9)$. However, the antitumor effect of HDAC inhibitor on MEC 
A
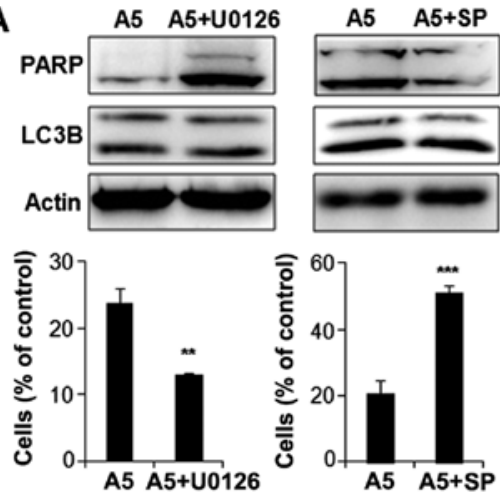

B

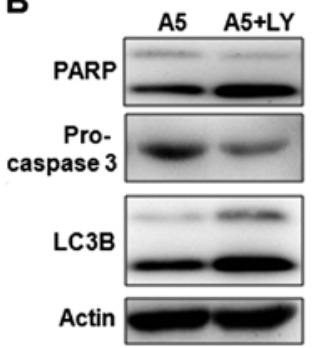

C
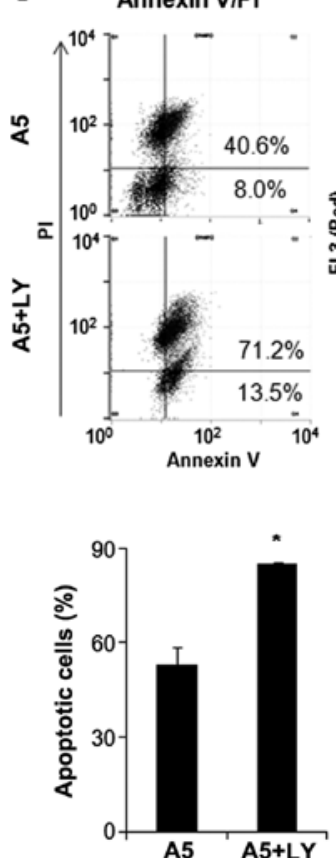

Acridine orange
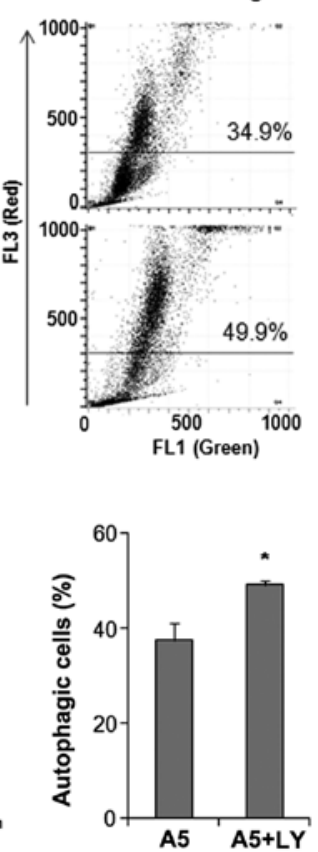

Figure 7. Effect of specific kinase inhibitors on apicidin-induced cell death in YD-15 cells. (A) The cells were treated with $5 \mu \mathrm{M}$ apicidin (A5) after pretreatment of U0126 $10 \mu \mathrm{M}$ (ERK inhibitor) or in the presence of SP600125 $10 \mu \mathrm{M}$ (SP, JNK inhibitor). Western blot analysis was performed using PARP and LC3B. The protein levels were normalized by a comparison with actin levels. Cell viability was determined by trypan blue exclusion assay. (B) The cells were treated with $5 \mu \mathrm{M}$ apicidin after pretreatment of LY294002 $50 \mu \mathrm{M}$ (LY, PI3K/AKT inhibitor). Western blot analysis was performed using PARP, pro-caspase-3 and LC3B. The protein levels were normalized by a comparison with the $\beta$-actin levels. Cell viability was determined using a trypan blue exclusion assay. (C) Apoptosis was determined by flow cytometry for Annexin V-FITC and PI dual labeling. Autophagy was determined by flow cytometry using acridine orange staining. The data are presented as the mean \pm SD of three independent experiments. * $\mathrm{P}<0.05$ compared to the untreated control. JNK, c-Jun NH2-terminal kinase; ERK, extracellular signal-regulated kinase; PARP, poly ADP ribose polymerase; LC3B, light change 3B; PI, propidium iodide.

A

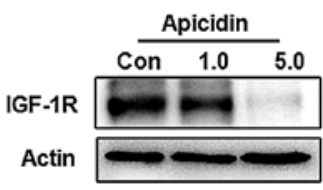

$\mathbf{B}$
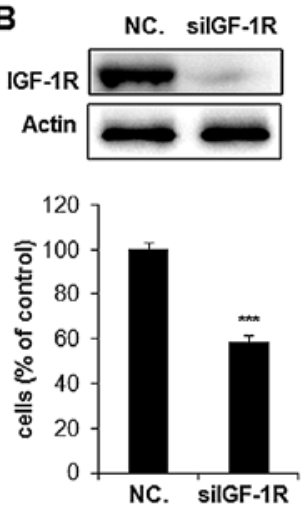

C
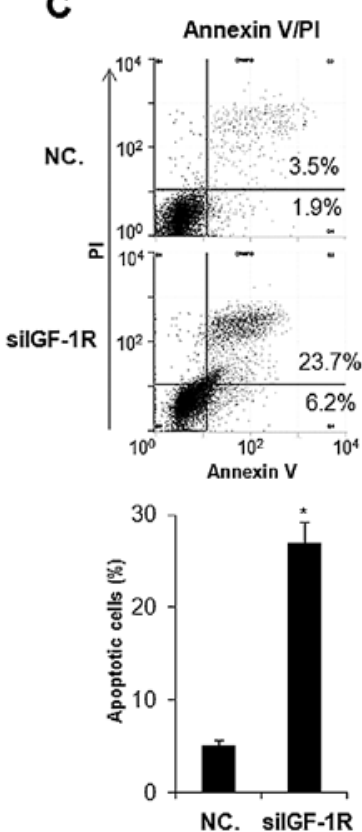

Acridine orange
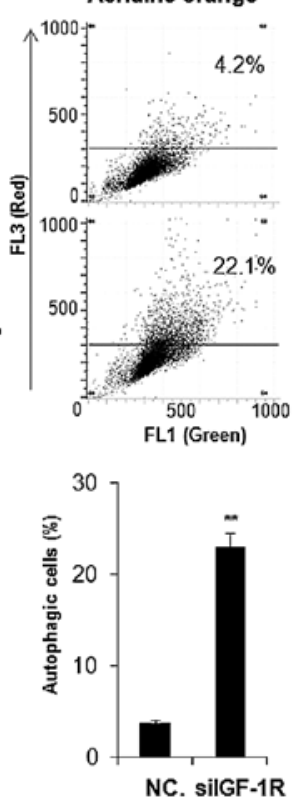

D

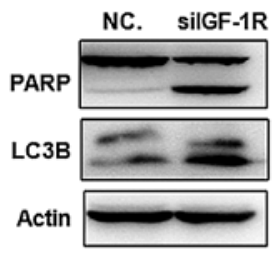

E

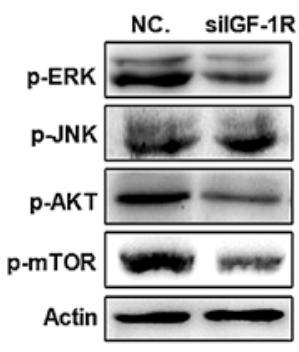

Figure 8. Inhibitory effect of IGF-1R by apicidin and IGF-1R siRNA transfection in YD-15 cells. (A) Expression of IGF-1R in apicidin-treated YD-15 cells. The cells were treated with apicidin $(1.0$ and $5.0 \mu \mathrm{M})$ for $48 \mathrm{~h}$. Cell lysates were subjected to western blot analysis using an antibody against IGF-1R. The protein levels were normalized by by a comparison with the actin levels. (B) The cells were transfected with IGF-1R siRNA (100 nM) and NC. siRNA (100 nM). After $24 \mathrm{~h}$ transfection, the medium was changed to remove the transfection complexes and the cells were harvested after $48 \mathrm{~h}$. Western blot analysis was performed using IGF-1R. The protein levels were normalized by a comparison with the actin levels. Cell viability was determined using a trypan blue exclusion assay. (C) Apoptosis was determined by flow cytometry for Annexin V-FITC and PI dual labeling. Autophagy was determined by flow cytometry using acridine orange staining. The data are presented as the mean $\pm \mathrm{SD}$ of three independent experiments. ${ }^{* * *} \mathrm{P}<0.01,{ }^{* * * * *} \mathrm{P}<0.001$, compared to the untreated control. (D) Western blot analysis was performed using PARP and LC3B. The protein levels were normalized by comparing to the actin levels. (E) Western blot analysis was performed using p-ERK, p-JNK, p-AKT and p-mTOR. The protein levels were normalized by a comparison with the actin levels. The representative results from three independent experiments are shown. IGF-1R, insulin-like growth factor 1 receptor; PARP, poly ADP ribose polymerase; LC3B, light change 3B; ERK, extracellular signal-regulated kinase; JNK, c-Jun NH2-terminal kinase; PI, propidium iodide. 
has yet to be determined. The present study first examined the antitumor efficacy of apicidin against human MEC cells. The data showed that apicidin inhibited cell proliferation and HDAC activity in YD-15 cells. Inhibition of cell growth by apicidin was also confirmed with MC-3 human MEC cells (data not shown). Our previous study reported for the first time that apicidin induced both apoptosis and autophagy in OSCC cells (18). Consistent with this observation, apicidin induced apoptosis as well as autophagy in YD-15 cells.

Several studies have described autophagy in tumor cell lines treated with chemotherapeutic agents including HDAC inhibitor. However, whether this role is protective or toxic to cancer cells has to be answered differently depending on the model used $(1,26,27)$. Autophagy offers protection against cancer by promoting autophagic cell death or contributing to cancer by promoting the survival of nutrient-starved cells (28). Moreover, the relationship between apoptosis and autophagy is different in cancer cells depending on the type of cell stress induced; in some cases apoptosis and autophagy act antagonistically, while in other cases autophagy enables apoptosis (29-31). To determine the function of autophagy and the relationship between apoptosis and autophagy, an autophagy inhibitor, CQ, and caspase inhibitor, z-VAD-fmk, were treated with apicidin. The inhibition of autophagy by CQ reduced the cell viability through enhanced apicidininduced apoptotic cell death, similar to previous studies on apicidin-treated OSCC cells (18). By contrast, the inhibition of apoptosis by z-VAD-fmk increased apicidin-induced autophagy, resulting in an increase in cell survival. These results suggest that apoptosis and autophagy by apicidin act antagonistically and autophagy exerts a protective role towards the activation of apoptosis by apicidin treatment in human MEC cells.

Although apicidin induces apoptosis and autophagy in cancer cells, the molecular mechanisms of apicidin remain unclear. The MAPK and PI3K/AKT/mTOR pathways are important signaling cascades in human cancer as a result of genetic alterations (32). The present results showed that apicidin inhibited the activation of ERK and AKT/mTOR but also induced JNK activation in YD-15 cells. Although JNK and p38 MAPK activities are involved in pro-apoptotic events, activation of the ERK pathway is involved in numerous cell responses, such as cell proliferation and survival (33). Similarly, the activated AKT pathway is involved in survival and proliferation (34). Inhibition of the MEK/ERK and AKT signaling pathways potentiates HDAC inhibitor-induced cell death in cancer cells $(35,36)$. Therefore, we suggest that apicidin inhibits cell proliferation through the inactivation of ERK, AKT signals and activation of JNK signal. It was confirmed that ERK and AKT inhibitors enhanced apicidin-induced cytotoxicity, whereas JNK inhibitors reduced this cytotoxicity. This result suggests that apicidininduced cell death is mediated by the reduction of ERK and AKT, and induction of JNK pathways in YD-15 cells.

The present study aimed to identify which signaling pathway is involved in apicidin-induced apoptosis and autophagy. The MAPK and PI3K/AKT/mTOR signaling pathways are well characterized with regard to the transmission of anti-apoptotic signals in cell survival and determining the effect of apoptotic responses on anticancer drugs $(37,38)$.
Disruption of the PI3K/AKT signaling pathway, culminating in the inhibition of AKT has been consistently associated with indicators of autophagy in cancer cells $(39,40)$. Inhibition of AKT signaling reduces the activity of mTOR, which is a key negative regulator of autophagy. The MAPK/ERK signaling pathway has been associated with the regulation of autophagy. In particular, the upregulation of ERK1/2 activity is imperative for induction of autophagy in cancer cells (41). Our results show that the inhibition of ERK and JNK activation oppositely regulated apicidin-induced apoptosis, although the autophagic effect was unaffected. By contrast, the inhibition of AKT activation enhanced apicidin-induced apoptosis and autophagic effects. Therefore, the data suggest that the apoptotic effect of apicidin is mediated by the ERK, JNK and AKT/mTOR pathways, whereas the autophagic response of apicidin is mediated by the AKT/mTOR pathways.

We also aimed to identify a potential upstream regulator of MAPK and AKT/mTOR pathways modulated by apicidin. The present study focused on IGF-1R, which is one of the upstream receptor tyrosine kinase responsible for activating the two major cascades, PI3K/AKT and MAPK (22). IGF-1R is frequently overexpressed in several cancers and the tumor-genetic salivary gland epithelial cells model and is now an attractive anticancer treatment target $(42,43)$. Previous studies have shown that HDAC inhibitors inhibited IGF-1R expression in cancer cells $(44,45)$. The present data also show that apicidin markedly inhibited the level of IGF-1R expression in YD-15 cells. Inhibition of IGF-1R expression by apicidin was also confirmed with MC-3 human MEC cells (data not shown). To further examine whether the inhibition of IGF-1R regulates apoptosis and autophagy through MAPK and AKT/mTOR pathway, IGF-1R was silenced by siRNA transfection in YD-15 cells. The data show that silencing of IGF-1R with siRNA results in apoptosis and autophagy through the action of downstream kinases, and is similar to the results obtained by apicidin treatment in YD-15 cells. These results suggest that apicidin exerts its antiproliferative properties through the suppression of IGF-1R in MEC cells.

In conclusion, apicidin induces apoptotic cell death by inactivating the ERK and AKT/mTOR signaling pathways and activating JNK signaling, whereas apicidin promoted pro-survival autophagy by inactivating the AKT/mTOR signaling pathways in YD-15 cells. These two events are mediated by the inhibition of IGF-1R. Therefore, the present study suggests that apicidin is an attractive antitumor agent for MEC treatment and a good candidate for targeting IGF-1R for cancer therapy. In addition, autophagy inhibitor enhances the anti-therapeutic effects of apicidin against human MEC.

\section{Acknowledgements}

This study was supported by the Basic Science Research Program through the National Research Foundation of Korea (NRF) funded by the Ministry of Education, Science and Technology (NRF-2011-0023907, NRF-2013R1A2A2A01067254).

\section{References}

1. Kim HJ and Bae SC: Histone deacetylase inhibitors: molecular mechanisms of action and clinical trials as anti-cancer drugs. Am J Transl Res 3: 166-179, 2011. 
2. Grunstein M: Histone acetylation in chromatin structure and transcription. Nature 389: 349-352, 1997.

3. Miller CP, Singh MM, Rivera-Del Valle N, Manton CA and Chandra J: Therapeutic strategies to enhance the anticancer efficacy of histone deacetylase inhibitors. J Biomed Biotechnol 2011: 514261, 2011.

4. Carew JS, Giles FJ and Nawrocki ST: Histone deacetylase inhibitors: mechanisms of cell death and promise in combination cancer therapy. Cancer Lett 269: 7-17, 2008.

5. Spiegel S, Milstien S and Grant S: Endogenous modulators and pharmacological inhibitors of histone deacetylases in cancer therapy. Oncogene 31: 537-551, 2012.

6. Jung M: Inhibitors of histone deacetylase as new anticancer agents. Curr Med Chem 8: 1505-1511, 2001.

7. Darkin-Rattray SJ, Gurnett AM, Myers RW, et al: Apicidin: a novel antiprotozoal agent that inhibits parasite histone deacetylase. Proc Natl Acad Sci USA 93: 13143-13147, 1996.

8. Ahn MY, Na YJ, Lee JW, Lee BM and Kim HS: Apicidin induces apoptosis via Cytochrome c-mediated intrinsic pathway in human ovarian cancer cells. Biomol Ther 17: 17-24, 2009.

9. Im JY, Park H, Kang KW, Choi WS and Kim HS: Modulation of cell cycles and apoptosis by apicidin in estrogen receptor (ER)-positive and-negative human breast cancer cells. Chem Biol Interact 172: 235-244, 2008.

10. Ahn MY, Lee J, Na YJ, Choi WS, Lee BM, Kang KW and Kim HS: Mechanism of apicidin-induced cell cycle arrest and apoptosis in Ishikawa human endometrial cancer cells. Chem Biol Interact 179: 169-177, 2009.

11. Choi KH, Shim JH, Huong LD, Cho NP and Cho SD: Inhibition of myeloid cell leukemia-1 by tolfenamic acid induces apoptosis in mucoepidermoid carcinoma. Oral Dis 17: 469-475, 2011

12. Shen S, Kepp O, Michaud M, et al: Association and dissociation of autophagy, apoptosis and necrosis by systematic chemical study. Oncogene 30: 4544-4556, 2011.

13. Portt L, Norman G, Clapp C, Greenwood M and Greenwood MT: Anti-apoptosis and cell survival: a review. Biochim Biophys Acta 1813: 238-259, 2011

14. Janku F, McConkey DJ, Hong DS and Kurzrock R: Autophagy as a target for anticancer therapy. Nat Rev Clin Oncol 8: 528-539, 2011.

15. Carew JS, Nawrocki ST and Cleveland JL: Modulating autophagy for therapeutic benefit. Autophagy 3: 464-467, 2007.

16. Rikiish H: Autophagic and apoptotic effects of HDAC inhibitors on cancer cells. J Biomed Biotechnol 2011: 830260, 2011.

17. Amaravadi RK and Thompson DB: The roles of therapy-induced autophagy and necrosis in cancer treatment. Clin Cancer Res 13: 7271-7279, 2007

18. Ahn MY, Ahn SG and Yoon JH: Apicidin, a histone deaceylase inhibitor, induces both apoptosis and autophagy in human oral squamous carcinoma cells. Oral Oncol 47: 1032-1038, 2011.

19. Lee EJ, Kim J, Lee SA, Kim EJ, Chun YC, Ryu MH and Yook JI: Characterization of newly established oral cancer cell lines derived from six squamous cell carcinoma and two mucoepidermoid carcinoma cells. Exp Mol Med 37: 379-390, 2005.

20. Kanematsu S, Uehara N, Miki H, Yoshizawa K, Kawanaka A Yuri $\mathrm{T}$ and Tsubura A: Autophagy inhibition enhances sulforaphane-induced apoptosis in human breast cancer cells. Anticancer Res 30: 3381-3390, 2010.

21. Warnken M, Reitzenstein U, Sommer A, et al: Characterization of proliferative effects of insulin, insulin analogues and insulin-like growth factor-1 (IGF-1) in human lung fibroblasts. Naunyn Schmiedebergs Arch Pharmacol 382: 511-524, 2010.

22. Brunet A and Pouyssegur J: Mammalian MAP kinase modules: how to transduce specific signals. Essays Biochem 32: 1-16, 1997

23. LeRoith D and Roberts CT Jr: The insulin-like growth factor system and cancer. Cancer Lett 195: 127-137, 2003.

24. Lalami Y, Vereecken P, Dequanter D, Lothaire P and Awada A: Salivary gland carcinomas, paranasal sinus cancers and melanoma of the head and neck: an update about rare but challenging tumors. Curr Opin Oncol 18: 258-265, 2006.

25. McHugh CH, Roberts DB, El-Naggar AK, et al: Prognostic factors in mucoepidermoid carcinoma of the salivary glands. Cancer 118: 3928-3936, 2012.
26. Kanzawa T, Germano IM, Komata T, Ito H, Kond Y and Kondo S: Role of autophagy in temozolomide-induced cytotoxicity for malignant glioma cells. Cell Death Differ 11: 448-457, 2004.

27. Shao Y, Gao Z, Marks PA and Jiang X: Apoptotic and autophagic cell death induced by histone deacetylase inhibitors. Proc Natl Acad Sci USA 101: 18030-18035, 2004.

28. Liu YL, Yang PM, Shun CT, Wu MS, Weng JR and Chem CC: Autophagy potentiates the anti-cancer effects of the histone deacetylase inhibitors in hepatocellular carcinoma. Autophagy 6 : 1057-1065, 2010.

29. Amaravadi RK, Yu D, Lum JJ, et al: Autophagy inhibition enhances therapy-induced apoptosis in a Myc-induced model of lymphoma. J Clin Invest 117: 326-336, 2007.

30. Boya P, Gonzalez-Polo RA, Casares N, et al: Inhibition of macroautophagy triggers apoptosis. Mol Cell Biol 25: 1025-1040, 2005

31. Yan $\mathrm{CH}$, Liang ZQ, Gu ZL, Yang YP, Reid $\mathrm{P}$ and Qin $\mathrm{ZH}$ Contributions of autophagic and apoptotic mechanisms to CrTX-induced death of K562 cells. Toxicon 47: 521-530, 2006.

32. De Luca A, Maiello MR, D'Alessio A, Pergameno $M$ and Normanno N: The RAS/RAF/MEK/ERK and the PI3K/AKT signalling pathways: role in cancer pathogenesis and implications for therapeutic approaches. Expert Opin Ther Targets 16: S17-27, 2012.

33. Yu C, Friday BB, Lai JP, McCollum A, Atadja P, Roberts LR and Adjei AA: Abrogation of MAPK and Akt signaling by AEE788 synergistically potentiates histone deacetylase inhibitor-induced apoptosis through reactive oxygen species generation. Clin Cancer Res 13: 1140-1148, 2007.

34. Sarker D, Reid AH, Yap TA and de Bono JS: Targeting the PI3K/AKT pathway for the treatment of prostate cancer. Clin Cancer Res 15: 4799-4805, 2009.

35. Jang ER, Kim YJ, Myung SC, Kim W and Lee CS: Different effect of protein kinase B/Akt and extracellular signal-regulated kinase inhibition on trichostatin A-induced apoptosis in epithelial ovarian carcinoma cell lines. Mol Cell Biochem 353: 1-11, 2011.

36. Nishioka C, Ikezoe T, Yang J, Koeffler HP and Yokoyama A: Inhibition of MEK/ERK signaling synergistically potentiates histone deacetylase inhibitor-induced growth arrest, apoptosis and acetylation of histone $\mathrm{H} 3$ on p21waf1 promoter in acute myelogenous leukemia cell. Leukemia 22: 1449-1452, 2008

37. Kennedy SG, Wagner AJ, Conzen SD, Jordan J, Bellacosa A, Tsichlis PN and Hay N: The PI 3-kinase/Akt signaling pathway delivers an antiapoptotic signal. Genes Dev 11: 701-713, 1997.

38. Widmann C, Gibson S, Jarpe MB and Johnson GL: Mitogenactivated protein kinase: conservation of a three-kinase module from yeast to human. Physiol Rev 79: 143-180, 1999.

39. Takeuchi H, Kanzawa T, Kondo $Y$ and Kondo S: Inhibition of platelet-derived growth factor signaling induces autophagy in malignant glioma cells. Br J Cancer 90: 1069-1075, 2004.

40. Mochizuki T, Asai A, Saito N, et al: Akt protein kinase inhibits non-apoptotic programmed cell death induced by ceramide. J Biol Chem 277: 2790-2797, 2002.

41. Ellington AA, Berhow MA and Singletary KW: Inhibition of Akt signaling and enhanced ERK1/2 activity are involved in induction of macroautophagy by triterpenoid B-group soyasaponins in colon cancer cells. Carcinogenesis 27: 298-306, 2006.

42. Riedemann J and Macaulay VM: IGF1R signalling and its inhibition. Endocr Relat Cancer 13: S33-43, 2006.

43. Obara K, Ide F, Mishima K, Inoue H, Yamada H, Hayashi Y and Saito I: Biological and oncogenic properties of p53-deficient salivary gland epithelial cells with particular emphasis on stromal-epithelial interactions in tumorigenesis. Pathobiology 73 : 261-270, 2006

44. Mitsiades CS, Mitsiades NS, McMullan CJ, et al: Transcriptional signature of histone deacetylase inhibition in multiple myeloma: biological and clinical implications. Proc Natl Acad Sci USA 101: 540-545, 2004.

45. Kim SO, Choi BT, Choi IW, et al: Anti-invasive activity of histone deacetylase inhibitors via the induction of Egr-1 and the modulation of tight junction-related proteins in human hepatocarcinoma cells. BMB Rep 42: 655-660, 2009. 